\title{
Exoplanet Transit Spectroscopy of Hot Jupiters Using HST/WFC3
}

\author{
Korey Haynes ${ }^{1,2,7}$, Avi M. Mandell ${ }^{1}$, Evan Sinukoff ${ }^{3}$, Nikku \\ Madhusudhan $^{4}$, Adam Burrows ${ }^{5}$ and Drake Deming ${ }^{6}$ \\ ${ }^{1}$ Solar System Exploration Division, NASA Goddard Space Flight Center, Greenbelt, MD \\ 20771, USA \\ ${ }^{2}$ Department of Physics and Astronomy, George Mason University, Fairfax, VA 22030, USA \\ ${ }^{3}$ Institute for Astronomy, University of Hawaii, Honolulu, HI 96822, USA \\ ${ }^{4}$ Department of Astronomy, University of Maryland, College Park, MD 20742, USA \\ ${ }^{5}$ Yale Center for Astronomy and Astrophysics, Yale University, New Haven, CT 06511, USA \\ ${ }^{6}$ Department of Astrophysical Sciences, Princeton University, Princeton, NJ 08544, USA \\ ${ }^{7}$ Corresponding Email: Korey.N.Haynes@nasa.gov
}

\begin{abstract}
We present analysis of transit spectroscopy of three extrasolar planets, WASP-12 b, WASP-17 b, and WASP-19 b, using the Wide Field Camera 3 (WFC3) on the Hubble Space Telescope (HST). Measurement of molecular absorption in the atmospheres of these planets offers the chance to explore several outstanding questions regarding the atmospheric structure and composition of these highly irradiated, Jupiter-mass objects. We analyze the data for a single transit for each planet, using a strategy similar in certain aspects to the techniques used by Berta (2012), and achieve almost photon-limited results for individual spectral bins. Our final transit spectra are consistent with the presence of a broad absorption feature at $1.4 \mu \mathrm{m}$ most likely due to water, but the amplitude of the absorption is less than expected based on previous observations with Spitzer, possibly due to hazes absorbing in the NIR. However, the degeneracy of models with different compositions and temperature structures combined with the low amplitude of any features in the data preclude our ability to place unambiguous constraints on the atmospheric composition without a comprehensive multi-wavelength analysis.
\end{abstract}

Keywords. stars: planetary systems, transits, techniques: photometric, techniques: spectroscopic

\section{Introduction}

The Wide Field Camera 3 (WFC3) on the Hubble Space Telescope (HST) provides the capability for spectroscopic characterization of molecular features in exoplanet atmospheres. WFC3 is an optical/NIR camera with the capability for slitless grism spectroscopy, with wavelength coverage in the the IR spanning between 0.8 and $1.7 \mu \mathrm{m}$ (Dressel (2012)). This region spans both the major bands of water between 1.3 and $1.5 \mu \mathrm{m}$ as well as another water band at $1.15 \mu \mathrm{m}$, and bands of a few other molecular species. Observations measuring flux within NIR water bands are impossible from the ground due to the extinction and variability caused by water vapor in the Earth's atmosphere; WFC3 therefore represents the only current platform for measuring absorption and/or emission from water in exoplanet atmospheres.

We present WFC3 observations of three transiting "hot Jupiter" exoplanets - WASP-12 b, WASP-17 b, and WASP-19 b - during transit of the host star. Two of these data sets, WASP-17 b and WASP-19 b, were observed as part of a large HST program to examine single transits and eclipses from a number of hot Jupiters (P.I. D. Deming), while the 
Table 1. Observing details for all three targets. Note that larger subarray size and higher peak pixel values correspond to larger peak to peak systematics.

\begin{tabular}{|l|c|c|c|}
\hline & WASP-12 & WASP-17 & WASP-19 \\
\hline Date of Observation & 2011-04-12 & 2011-07-08 & 2011-07-01 \\
\hline Integration Time (seconds) & 7.624 & 12.795 & 21.657 \\
\hline Subarray Mode (pix $\left.{ }^{2}\right)$ & 256 & 512 & 128 \\
\hline CALWF3 version & 2.7 & 2.3 & 2.3 \\
\hline NSamp & 3 & 16 & 5 \\
\hline Timing Sequence & SPARS10 & RAPID & SPARS10 \\
\hline $\begin{array}{l}\text { Peak Pixel Value (counts) } \\
\text { Peak to Peak Systematics }\end{array}$ & 38,000 & 64,000 & 73,000 \\
\hline $\begin{array}{l}\text { Range (Combined Light) } \\
\left(10^{-3} \text { Normalized Flux) }\right.\end{array}$ & 0.80 & 3.94 & 1.62 \\
\hline
\end{tabular}

data for the transit of WASP-12 b were taken as part of a single-object campaign (P.I. M. Swain) and first analyzed in Swain et al. (2013). Observational details are listed in Table 1. All three planets orbit extremely close to their parent star, are therefore highly irradiated, and have large atmospheric scale heights, making them excellent targets for transmission spectroscopy.

Recent observational studies have produced conflicting results regarding the atmospheric compositions of several hot Jupiters, including WASP-12 b and WASP-19 b. Madhusudhan et al. (2011) first raised the possibility of a non-solar abundance in the atmosphere of WASP-12 b using occultation measurements in four Spitzer photometric bands (Campo et al. 2011) and three ground-based NIR photometric bands Croll et al. (2011) to constrain the carbon-to-oxygen ratio to super-solar values, possibly greater than unity. Similar Spitzer and ground-based measurements for WASP-19 b were consistent with both solar and super-solar C/O models (Anderson et al. 2011), raising the possibility of a population of carbon-rich hot Jupiters. However, Crossfield et al. (2012) recently re-analyzed the Spitzer data for WASP-12 b in light of the discovery of a faint candidate companion imaged by Bergfors et al. (2012), concluding that the dilutioncorrected Spitzer and ground-based photometry can be fit by solar-metallicity models with almost isothermal temperature structures.

\section{Data Reduction and Analysis}

WFC3 data show strong systematics, first described by Berta et al. (2012). Swain et al. (2013) examines the systematics from multiple observing campaigns, and determines that the systematics are mainly influenced by the maximum exposure levels and choice of subarray size. In order to remove these systematics, we employ the divide-oot method described by Berta et al. (2012), averaging the out-of-transit, combined-light data to create a template for the systematics, the scaling of which we leave as an open parameter when fitting our channel light curves (see Figure 1). For fitting purposes, we use a Markov Chain Monte Carlo (MCMC) routine with a Metropolis-Hastings sampler Ford (2005), using the light curve model from Mandel \& Agol (2002), with additional terms to account for the gradual decrease in flux seen in all WFC3 exoplanet transit data to date, the systematics scaling term, as well as two further scaling terms to account for shifts on the 


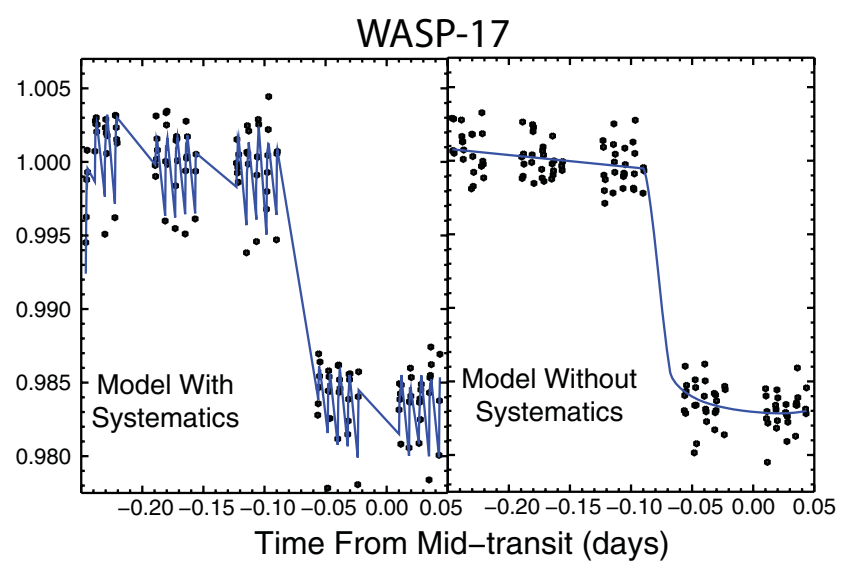

Figure 1. Sample bin for WASP-17 showing the data (black) with model (blue) including systematics (left), and with systematics removed (right).

detector in position and wavelength space. Systematics scaling terms are allowed to vary only if the resulting fit has a $\Delta \mathrm{BIC} \geqslant 2$,

We also take care to remove contamination due to WASP-12's recently discovered companion, Bergfors-6 (Bergfors et al. 2012). We do this by using WASP-19's PSF as a template for an uncontaminated source. We determine the positions of WASP-12 and Bergfors- 6 in the direct image, place one PSF at the location of each source, and then jointly scale the heights of each using a $\chi^{2}$ fitting routine.

\section{Results}

In Figure 2 we show the resulting spectra for each planet and overplot several models based on the framework of Burrows et al. (2000) (and more recently Burrows et al. (2006), Burrows et al. (2008) and Howe \& Burrows (2012) and Madhusudhan \& Seager (2009) and Madhusudhan et al. (2011). The Burrows models calculate the chemical and radiative equilibrium state of each planet based on the mass, size, and incident radiation, assuming solar abundances. For WASP-17, the data is well fit by either a standard model or a hazy, isothermal model, while the data for WASP-12 b is consistent within uncertainties with a flat spectrum.

The Madhusudhan models relax the stringent requirements for radiative and chemical equilibrium, instead exploring oxygen-rich or a carbon-rich chemistry at a specific temperatures (see Madhusudhan 2012 for details). For WASP-19 b the carbon-rich models fit the data slightly better, but we are unable to discriminate between the models based solely on this data.

Huitson et al. (2013) have also analyzed the same WASP-19 b data set, and find similar results to our data. Swain et al. (2013) and Stevenson et al. (2013) have both analyzed the WASP-12 b data, and find a deeper transit depth at the short wavelength edge of the spectrum.

We conclude that the data can be fit with standard atmospheric models, without the need for exotic chemistry or temperature structure. Models with a deep absorption feature are ruled out by our data. However, any of these models can still be adjusted to fit the data by adding an absorbing haze layer or decreasing the water abundance. A determination of the carbon-to-oxygen ratio cannot be deduced from our current data set alone because the location of absorption bands are similar in both oxygen-rich and 


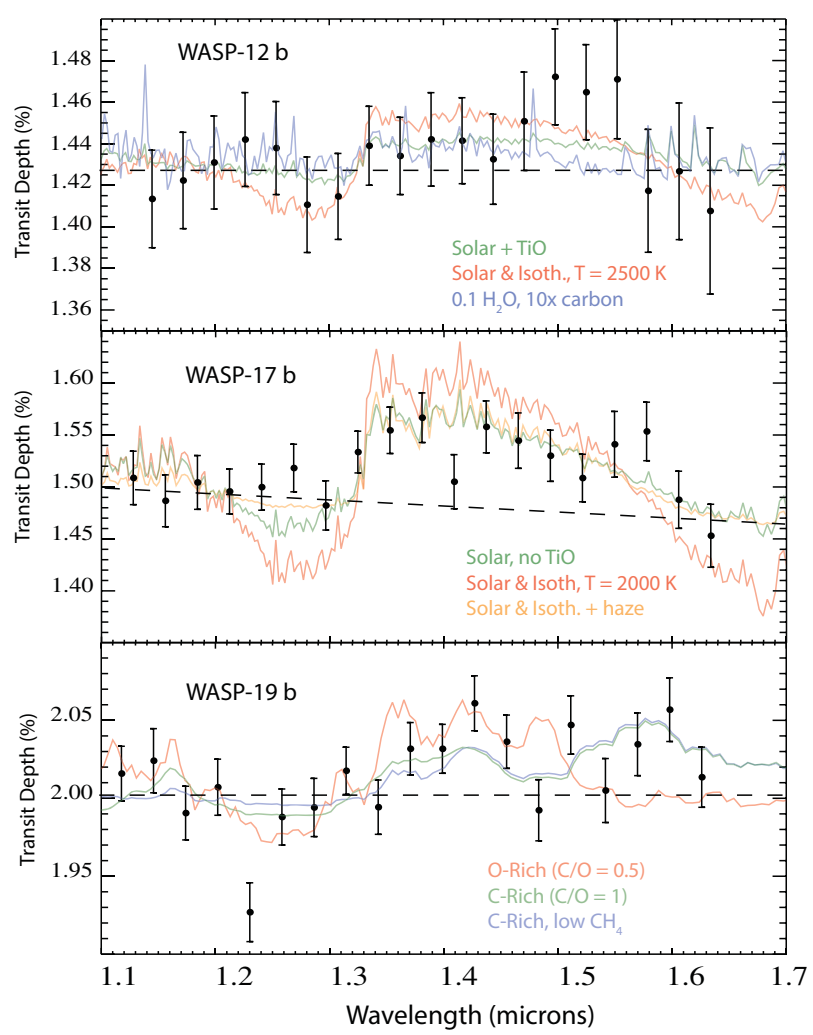

Figure 2. Transit depths for 19 bins for each target, with models from Burrows et al. (WASP-17 $\mathrm{b}$ and WASP-12 b) and Madhusudhan et al. (WASP-19 b). Models show suppressed water features, possibly indicating a haze layer. Data is undergoing further analysis.

carbon-rich models. Similarly, confirmation of the presence or absence of haze requires an analysis of transit depth measurements taken across a wide wavelength range in both the optical and IR, and is beyond the scope of the current work.

\section{References}

Anderson, D. R., et al. 2011, MNRAS, 416, 2108

Bergfors, C., et al. 2012, MNRAS, 428, 182

Berta, Z. K., et al. 2012, ApJ, 747, 35

Burrows, A., Marley, M. S., \& Sharp, C. M. 2000, ApJ, 531, 438

Burrows, A., Sudarsky, D., \& Hubeny, I. 2006, ApJ, 650, 1140

Burrows, A., Budaj, J., \& Hubeny, I. 2008, ApJ, 678, 1436

Campo, C. J., et al. 2011, ApJ, 727, 125

Croll, B., et al. 2011, ApJ, 141, 30

Crossfield, I. J. M., et al. 2012, ApJ, 760, 140

Dressel, L. 2012, "Wide Field Camera 3 Instrument Handbook, Version 5.0" (Baltimore: STScI)

Ford, E. B. 2005, ApJ, 129,1706

Howe, A. R. \& Burrows, A. S. 2012, ApJ, 756,176

Huitson, C. M. et al. 2013, MNRAS, 434, 3252

Madhusudhan, N., \& Seager, S. 2009, ApJ, 707, 24

Madhusudhan, N., Mousis, O., Johnson, T. V., \& Lunine, J. I. 2011, ApJ, 743, 191

Madhusudhan, N. 2012, ApJ, 758, 36 
Mandel, K., \& Agol, E. 2002, ApJ, 580, L171

Stevenson, K. B. et al. 2013, arXiv:1305.1670

Swain. M., et al. 2013, Icarus, 225, 432

\section{Discussion}

TRIAUD: You mentioned that an offset between your results for WASP-12 and Swain's could be due to orbital uncertainties. Which parameters and what precision do you require as it might affect results in other parts, like Rayleigh scattering?

HAYNES: Many parameters can affect this. It is uncertain which are the most important but limb darkening is probably one. Maybe this should be taken more seriously but people have done mostly differential studies rather than absolute.

BARstow: Have you considered modeling the WFC3 systematics for removal?

HAYNES: This was one of our first approaches, but considering the short cadence of some of the systematics (only 5 exposures, in the case of WASP-17's hook/ramp effect), and the range of variables that affect the systematics (exposure time, total pixel illumination, sub-array size), we found the divide-oot method to produce much more reliable results.

BARstow: Could the high $\mathrm{C} / \mathrm{O}$ ratio for WASP-19 b also be explained by clouds or haze?

HAYNES: A haze layer or clouds remains a possibility, and there is no way to determine, from the available data, whether we see shallow water absorption features because there is truly little water, or because such features are obscured by haze. This is similar to the case of GJ1214 in Berta et al. (2012). 